\title{
Dynamique de mousses poroélastiques
}

\author{
Benjamin Sobac, Mathieu Colombani et Yö̈l Forterre ${ }^{a}$ \\ Laboratoire IUSTI CNRS UMR 6595, Aix-Marseille Université (I), 5 rue Enrico Fermi, 13453 Marseille Cedex 13, France
}

Reçu le 3 mai 2011, accepté le 23 mai 2011

\begin{abstract}
Résumé - Nous étudions expérimentalement la dynamique de mousses élastiques (mousse ouverte flexible en polyuréthane) plongées dans un fluide visqueux. La mousse est initialement comprimée dans une direction puis brutalement relâchée. Le champ de vitesse de la mousse et la pression du fluide interstitiel (pression de pore) sont mesurés au cours de la décompaction du système. Pour des petites compactions initiales, on observe que la mousse relaxe exponentiellement vers son état d'équilibre, avec une dynamique diffusive en accord quantitatif avec les théories classiques de consolidation des sols. En revanche, pour des grandes compactions initiales, la dynamique de relaxation est inhomogène et se déroule à travers un front de décompaction. Un modèle diphasique simple permet de prédire semi-quantitativement les observations et relie l'existence du front au caractère fortement non-linéaire de la réponse mécanique de la mousse.
\end{abstract}

Mots clés : Poroélasticité / matériaux cellulaires / front

\begin{abstract}
On the dynamics of poroelastic foams. We experimentally investigate the dynamics of soft polyurethane open foams immersed in viscous fluids. The elastic foam is initially compressed in one direction and then suddenly released. The velocity field of the foam and the dynamic pressure of the interstitial fluid (pore pressure) are measured during the swelling of the foam. For small initial compactions, the foams relax exponentially to equilibrium with a diffusive dynamics in quantitative agreements with classical theories of soil consolidation. On the other hand, for large initial compactions, the dynamics is strongly inhomogeneous and occurs through a localized decompaction front. A simple two-fluids model semi-quantitatively predicts the observations and links the existence of a localized front to the highly non-linear mechanical response of the foam.
\end{abstract}

Key words: Poroelasticity / cellular solids / non-linear front

\section{Introduction}

De nombreuses situations industrielles, géophysiques ou biologiques mettent en jeu la dynamique d'un milieu poreux déformable saturé en fluide. Un exemple classique est celui de la déformation des sédiments ou des roches saturés en mécanique des sols [1]. On peut également citer l'utilisation de mousses pour amortir les chocs dans l'industrie automobile, la mécanique des bétons ou la modélisation des os et des tissus végétaux en biomécanique. Dans tous ces exemples, la déformation du milieux poreux s'accompagne d'un écoulement fluide qui applique des contraintes supplémentaires sur la matrice solide. Ce couplage entre déformation d'un milieu poreux et écoulement, ou poroélasticité, a donné lieu à

\footnotetext{
a Auteur pour correspondance :

yoel.forterre@polytech.univ-mrs.fr
}

une très importante littérature depuis les travaux pionniers de Terzaghi et Biot [1-4]. Il existe toutefois peu d'expériences sur des systèmes poroélastiques modèles qui permettent de tester les hypothèses fondamentales [5-7]. De plus, ces études s'intéressent surtout au régime des petites déformations (sols, os) et peu de travaux ont étudié la dynamique d'objets poroélastiques fortement déformés, pertinents dans certains contextes industriel ou biologique (mousse, tissus mous).

L'objectif de cet article est de revisiter les concepts classiques de poroélasticité à partir d'une expérience contrôlée utilisant des mousses élastiques molles (mousse polyurethane flexible) plongées dans un fluide visqueux (huile silicone). Ces matériaux présentent une structure ouverte proche de celle d'une mousse liquide dont on aurait supprimé les films de Plateau (Fig. 1a). L'intérêt de ces matériaux pour notre étude est qu'ils possèdent 


\section{Nomenclature}

\begin{tabular}{|c|c|}
\hline$\nu$ & Coefficient de Poisson (drainé) de la mousse en compression dans le régime élastique linéaire \\
\hline$D(\phi)$ & Coefficient de diffusion non-linéaire défini par $D(\phi)=\frac{\phi k(\phi)(d \sigma(\phi) / d \phi)}{\eta}\left(\mathrm{m}^{2} \cdot \mathrm{s}^{-1}\right)$ \\
\hline$D_{1}$ & Coefficient de diffusion non-linéaire pour $\epsilon<\epsilon_{\mathrm{c}}\left(\mathrm{m}^{2} . \mathrm{s}^{-1}\right)$ \\
\hline$D_{2}$ & Coefficient de diffusion non-linéaire pour $\epsilon>\epsilon_{\mathrm{c}}\left(\mathrm{m}^{2} \cdot \mathrm{s}^{-1}\right)$ \\
\hline$p(z, t)$ & Champs de pression dynamique du fluide interstitiel ou pression de pore $(\mathrm{Pa})$ \\
\hline & C'est la pression moins la pression hydrostatique \\
\hline$\Delta H=H_{0}-H$ & Compression absolue de la mousse (définie positive) \\
\hline$\epsilon=\Delta H / H_{0}$ & Compression relative - ou déformation - de la mousse (définie positive) \\
\hline$\epsilon_{i}$ & Compression relative initiale $(t=0)$ de la mousse (définie positive) \\
\hline$\epsilon_{\mathrm{c}}$ & $\begin{array}{l}\text { Compression relative critique marquant la transition entre le régime de compression } \\
\text { linéaire et non-linéaire de la mousse }\end{array}$ \\
\hline$\sigma$ & Contrainte élastique dans la mousse, définie positive en compression $(\mathrm{Pa})$ \\
\hline$k_{n}$ & $\begin{array}{l}\text { Échelle spatiale caractéristique (inverse) du mode de perturbation de pression } n \text { dans le régime } \\
\text { linéaire donnée par } k_{n}=\left(\frac{\pi}{2 H}+\frac{n \pi}{H}\right)\left(\mathrm{m}^{-1}\right)\end{array}$ \\
\hline$\phi$ & Fraction volumique solide de la mousse \\
\hline$\phi_{0}$ & Fraction volumique au repos de la mousse (non déformée) \\
\hline$\phi_{i}$ & Fraction volumique initiale de la mousse compactée $(t=0)$ \\
\hline$\phi_{\mathrm{c}}$ & $\begin{array}{l}\text { Fraction volumique critique marquant la transition entre le régime de compression linéaire } \\
\text { et non-linéaire de la mousse }\end{array}$ \\
\hline$H(t)$ & Hauteur de la mousse à l'instant $t$ \\
\hline$H_{0}$ & Hauteur au repos de la mousse non déformée \\
\hline$n$ & Indice du mode de perturbation de pression dans le régime linéaire \\
\hline$\rho_{0}$ & Masse volumique au repos de la mousse $\left(\mathrm{kg} \cdot \mathrm{m}^{-3}\right)$ \\
\hline$\rho_{\mathrm{s}}$ & Masse volumique du solide composant le squelette de la mousse $\left(\mathrm{kg} \cdot \mathrm{m}^{-3}\right)$ \\
\hline$\rho_{\mathrm{f}}$ & Masse volumique du fluide interstitiel $\left(\mathrm{kg} . \mathrm{m}^{-3}\right)$ \\
\hline$E_{0}$ & Module de Young (drainé) de la mousse en compression dans le régime élastique linéaire $(\mathrm{Pa})$ \\
\hline$R e$ & Nombre de Reynolds à l'échelle du pore \\
\hline$E_{1}$ & Pente de la loi de comportement modélisée pour $\epsilon<\epsilon_{\mathrm{c}}(\mathrm{Pa})$ \\
\hline$E_{2}$ & Pente de la loi de comportement modélisée pour $\epsilon>\epsilon_{\mathrm{c}}(\mathrm{Pa})$ \\
\hline$k_{0}$ & Perméabilité hydraulique (Darcy) de la mousse non déformée $\left(\mathrm{m}^{2}\right)$ \\
\hline$k(\phi)$ & Perméabilité hydraulique (Darcy) de la mousse $\left(\mathrm{m}^{2}\right)$ \\
\hline$p_{i}$ & Pression de pore initiale dans la mousse juste après le relâchement de la mousse \\
\hline$t$ & Temps (s) $t=0$ correspond à l'instant où la mousse est libérée \\
\hline$\eta$ & Viscosité du fluide interstitiel (Pa.s) \\
\hline$V(z, t)$ & Vitesse locale de la mousse $\left(\mathrm{m} \cdot \mathrm{s}^{-1}\right)$ \\
\hline$v(z, t)$ & Vitesse locale du fluide $\left(\mathrm{m} . \mathrm{s}^{-1}\right)$ \\
\hline$\tau_{\mathrm{p}}$ & Temps poroélastique défini par $\tau_{\mathrm{p}}=(1-2 \nu) \eta H^{2} /(E k)$ (s) \\
\hline$\tau_{n}$ & $\begin{array}{l}\text { Temps caractéristique de décroissance du mode de perturbation de pression } n \\
\text { dans le régime linéaire donné par } \tau_{n}=\frac{\tau_{p}}{\left(\frac{\pi}{2}+n \pi\right)^{2}}(\mathrm{~s})\end{array}$ \\
\hline$z$ & $\begin{array}{l}\text { Variable spatiale dans la direction de compression }(\mathrm{m}) \cdot z=0 \text { correspond à la base } \\
\text { fixe de la mousse; } z=H \text { correspond à la surface libre de la mousse }\end{array}$ \\
\hline
\end{tabular}

un régime élastique (réversible) jusqu'aux grandes compressions. Leur réponse est de plus fortement non-linéaire avec l'apparition d'un plateau de contrainte au-delà d'une déformation critique $\epsilon_{\mathrm{c}}$ de l'ordre de $5 \%$ (Fig. 1a).

L'expérience que nous avons mise en place consiste à comprimer lentement une mousse confinée latéralement et plongée dans un fluide visqueux jusqu'à une déformation initiale donnée. On libère alors brutalement la contrainte extérieure. Sous l'effet des contraintes élastiques internes, la mousse se décompacte pour retrouver sa forme initiale. En retour, le fluide entre dans la mousse et exerce sur celle-ci une contrainte visqueuse de type Darcy qui s'oppose au mouvement. Dans le régime à bas nombre de Reynolds que nous étudions, l'inertie est négligeable et la dynamique de décompaction de la mousse est contrôlée par l'équilibre entre la contrainte élastique et la pression fluide.

Dans un premier temps, nous étudions la relaxation de la mousse pour des faibles compressions initiales (régime linéaire). Nous étudions ensuite le régime de décompaction pour des grandes compressions initiales (régime non-linéaire). Les résultats expérimentaux sont 

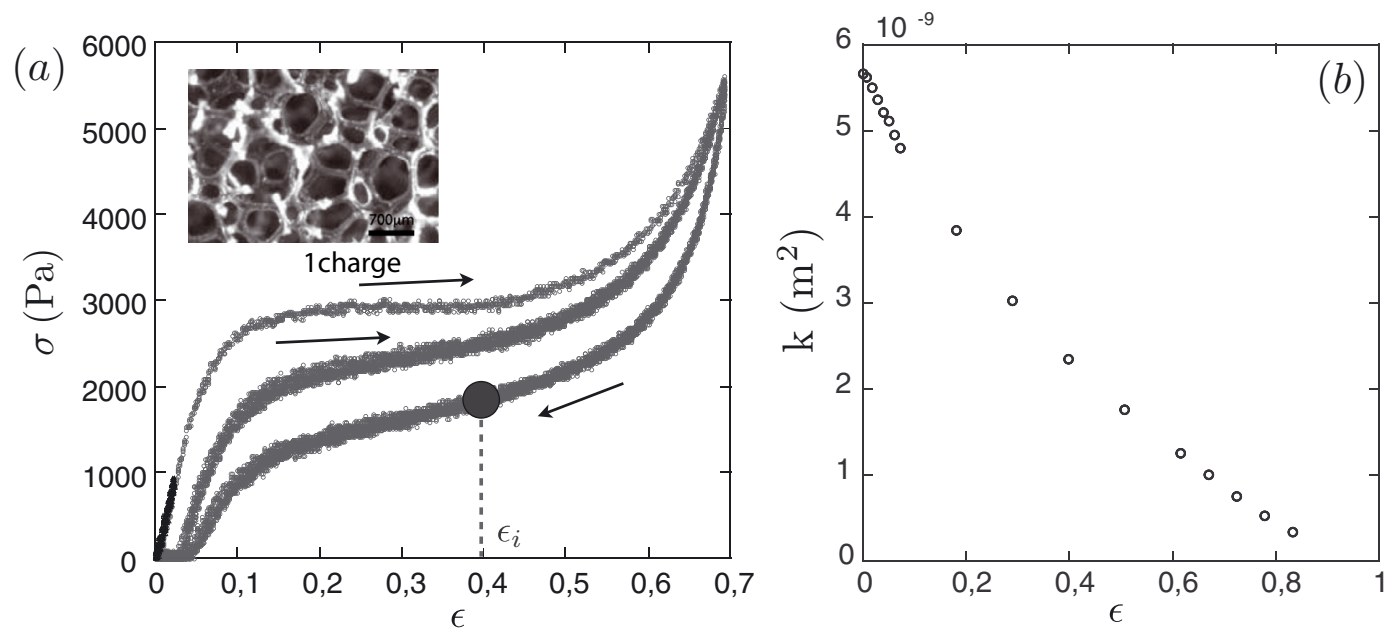

(c)

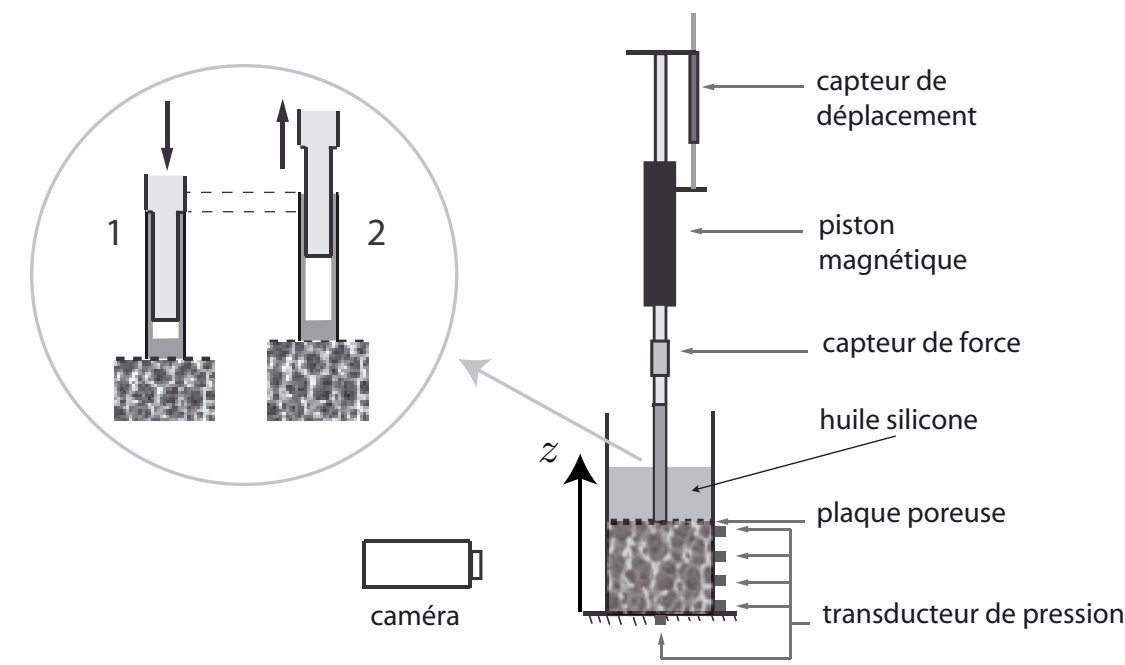

Fig. 1. (a) Comportement mécanique en compression des mousses étudiées (mesuré dans l'air). Dans les expériences, la compacité initiale est préparée en comprimant complètement la mousse jusqu'à $\epsilon=0,7$ puis en revenant vers $\epsilon_{\mathrm{i}}$. Avec ce protocole, la courbe de décharge est toujours la même. (b) Perméabilité hydraulique de la mousse en fonction de la compression $\epsilon=\Delta H / H_{0}$ où $H_{0}$ est la hauteur initiale de la mousse. (c) Dispositif expérimental d'étude de la dynamique d'une mousse élastique plongée dans un fluide visqueux.

confrontés à un modèle diphasique couplant la loi de comportement élastique de la mousse et la loi de Darcy.

\section{Matériels et méthodes}

\subsection{Mousses élastiques}

Les mousses élastiques utilisées sont des mousses flexibles en polyether à structure réticulée et cellules ouvertes, découpée à la taille voulue par le fournisseur (FoamPartner, Frina Mousse France, BP 55, 68272 Wittenheim France). La porosité des mousses est de 60 PPI (pore per inch), ce qui correspond à une taille de pore moyenne de $800 \mu \mathrm{m}$ (Fig. 1a, insert). La masse volumique moyenne des mousses est $\rho_{0}=30 \mathrm{~kg} \cdot \mathrm{m}^{-3}( \pm 2 \%)$. En prenant $\rho_{\mathrm{s}} \approx 1078 \mathrm{~kg} . \mathrm{m}^{-3}$ pour la masse volumique du polyether composant la mousse [7], on trouve que la fraction volumique des mousses est $\phi_{0}=\rho_{0} / \rho_{\mathrm{s}} \approx 0,03$. On utilise des blocs de mousses de base carrée de côté $a=8 \mathrm{~cm}$ et de hauteur $H_{0}=1,4 ; 2 ; 4 ; 6 ; 8 ; 12 \mathrm{~cm}$.

\subsection{Propriétés mécaniques des mousses en compression}

La loi de comportement en compression des mousses est mesurée à l'aide d'un banc mécanique composé d'un piston magnétique (STA2506S, Copley Controls Corp) muni d'un capteur de force $(0-500 \mathrm{~N}$, XFTC 300 et ARD 150, FGP Sensor \& Instrumentation) et de position (0-15 cm, TS 150 et MUP 100-1, FGP Sensor \& Instrumentation), l'ensemble étant interfacé sous Labview à l'aide d'une carte d'acquisition (USB-6009, National Instrument). Les mousses sont placées entre deux plaques rigides dans l'air et à bords libres. Un exemple de relation contrainte $(\sigma)$-déformation $(\epsilon)$ en compression 
est présenté sur la figure 1a pour 5 cycles de compression/décompression à $1 \mathrm{~mm} \cdot \mathrm{s}^{-1}$ entre $0<\epsilon<0,7$.

Pour $\epsilon<\epsilon_{\mathrm{c}} \approx 0,04$ (4\%), la relation contraintedéformation est linéaire et réversible, $\sigma=E_{0} \epsilon$, avec un module de Young $E_{0}=1,05 \times 10^{5} \mathrm{~Pa} \pm 30 \%$ (18 mesures, 2 échantillons). Dans ce régime, le coefficient de Poisson $\nu$ est défini à partir de la variation de volume de l'échantillon : $\Delta \mathcal{V} / \mathcal{V}_{0}=(1-2 \nu) \Delta H / H_{0}$, où $\mathcal{V}_{0}$ est le volume initial de la mousse et $\Delta \mathcal{V}$ (resp. $\Delta H$ ) la variation de volume (resp. hauteur) lors de la compression. Le volume est obtenu par visualisation de côté et traitement d'image (Nikon D300 et logiciel Image J) selon : $\mathcal{V} \approx \mathcal{A}^{2} / H$, où $\mathcal{A}$ est l'aire latérale de la mousse. On trouve $\nu=0,18 \pm 20 \%$. Notons que dans notre géométrie de compression uniaxiale à bords contraints, la contrainte n'est pas strictement homogène et le module de Young mesuré est en fait un module de Young apparent qui est fonction de la géométrie et du coefficient de Poisson du matériau [8]. Pour un facteur de forme $S=a /\left(4 H_{0}\right)$ et un coefficient de Poisson $\nu=0,18$, la résolution des équations de l'élasticité prédit une différence de seulement $2 \%$ entre le module apparent et le module de Young [8].

Pour $0<\epsilon<0,7$ (grandes compressions), on observe une forte hystérésis de la courbe charge-décharge, la première compression différant des cycles suivants. Audelà d'une compression critique, on observe un pseudoplateau de contrainte, correspondant au flambage des cellules qui constituent le matériau [9]. Enfin, pour des grandes compressions (>50-60\%), la contrainte remonte brutalement due à la densification du matériau.

\subsection{Caractérisation hydraulique des mousses : perméabilité}

La perméabilité hydraulique des mousses est déterminée en détectant la position $h(t)$ de l'interface lors de la vidange sous gravité d'un fluide visqueux qui traverse la mousse (huile silicone V47100 de viscosité $\eta=0,104$ Pa.s et densité $\left.\rho_{\mathrm{f}}=963 \mathrm{~kg} \cdot \mathrm{m}^{-3}\right)$. On a $h(t)=h_{0} \exp (-t / \tau)$ avec $\tau \propto \eta H_{0} /\left(k_{0} \rho_{\mathrm{f}} g\right)$. On trouve une perméabilité pour la mousse non déformée $k_{0}=4,95 \times 10^{-9} \pm 5 \% \mathrm{~m}^{2}$. Un piston à l'intérieur $\mathrm{du}$ tube de drainage permet d'étendre cette mesure pour des mousses comprimées ce qui permet de connaître la relation $k(\epsilon)$ ou $k(\phi)$ pour $0<\epsilon<0,85$ (Fig. 1b).

\subsection{Dynamique poroélastique : dispositif expérimental et protocole}

Le dispositif expérimental est présenté sur la figure 1c. Le bloc de mousse (base carrée $8 \mathrm{~cm} \times 8 \mathrm{~cm}$, hauteur $\left.H_{0}\right)$ est immergée dans une cuve en plexiglass $(8,3 \mathrm{~cm} \times 8,3 \mathrm{~cm} \times 16 \mathrm{~cm})$ remplie d'huile silicone (densité $\rho_{\mathrm{f}}=963 \mathrm{~kg} \cdot \mathrm{m}^{-3}$, viscosité $\eta=0,104$ Pa.s et $\eta=1$ Pa.s). Un piston magnétique relié à un capteur de force et de déplacement comprime la mousse verticalement par l'intermédiaire d'une grille poreuse. Un système de barreau coulissant permet de libérer brutalement la contrainte appliquée sans provoquer d'aspiration dans le fluide. 6 capteurs de pression (Data Instruments DCAL405DN, $\pm 76,2 \mathrm{~cm} \mathrm{H}_{2} \mathrm{O}$ ) situés sous la mousse et sur le côté permettent de mesurer la pression interstitielle du fluide (pression de pore). Enfin, un mouchetis est projeté sur les faces de la mousse pour détecter le champ de vitesse du solide par PIV (caméra Canon XM1).

Le protocole expérimental pour l'étude des petites déformations $\left(\epsilon<\epsilon_{\mathrm{c}}\right)$ est le suivant. La mousse subit tout d'abord 3 cycles complets de compression/décompression jusqu'à une déformation de $70 \%$. Puis la mousse est comprimée lentement (vitesse $1 \mathrm{~mm} . \mathrm{s}^{-1}$ ) jusqu'à une déformation initiale $\epsilon_{i}$ et cette déformation est maintenue pendant $7 \mathrm{~min}$. À $t=0$, le piston est remonté brutalement (vitesse $1 \mathrm{~m} . \mathrm{s}^{-1}$ ). Pendant la relaxation de la mousse vers son état d'équilibre, on mesure simultanément la pression interstitielle $p(z, t)$, la position de l'interface de la mousse $H(t)$ et le champ de vitesse de la mousse $V(z, t)$.

Le protocole expérimental pour l'étude des grandes déformations est un peu différent. La mousse est comprimée lentement (vitesse $0,3 \mathrm{~mm} . \mathrm{s}^{-1}$ ) jusqu'à une déformation de $70 \%$ puis décomprimée à la même vitesse jusqu'à la compression initiale voulue $\epsilon_{i}$. La contrainte est alors brutalement relâchée en remontant rapidement le piston $\left(1 \mathrm{~m} \cdot \mathrm{s}^{-1}\right)$. Ce protocole permet de s'affranchir des problèmes de vieillissement et d'hystérésis en sondant la même courbe de décharge $\sigma(\epsilon)$ quel que soit l'état initial $\epsilon_{i}$ (Fig. 1c).

\subsection{Méthode de résolution numérique}

Pour simuler l'équation de diffusion non-linéaire (5) avec les conditions aux limites (6), on utilise une méthode eulérienne par différences finies implémentée sous Matlab. La dérivée de la loi de comportement $\sigma^{\prime}(\phi)$ intervenant dans (5) est obtenue en modélisant la loi de comportement $\sigma(\epsilon)$ selon :

$$
\sigma^{\prime}(\epsilon)=E_{1}\left[1-\frac{1-\left(E_{2} / E_{1}\right)}{2}\left(1+\tanh \left(\frac{\epsilon-\epsilon_{\mathrm{c}}}{\delta}\right)\right)\right]
$$

avec $\epsilon=1-\left(\phi_{0} / \phi\right)\left(\phi_{0}=0,03\right.$ est la fraction volumique de la mousse au repos), où $\delta$ caractérise la taille de la transition entre le régime linéaire et le pseudo-plateau de contrainte.

\section{Résultats}

\subsection{Dynamique linéaire : poroélasticité}

Dans un premier temps, nous avons étudié la dynamique de décompaction d'une mousse d'épaisseur $H_{0}=$ $12 \mathrm{~cm}$ dans un fluide de viscosité $\eta=0,104$ pour une faible compression initiale $\epsilon_{\mathrm{i}}$, située dans le domaine d'élasticité linéaire $\left(\epsilon_{\mathrm{i}}<4 \%\right)$. Dès que la contrainte extérieure est 

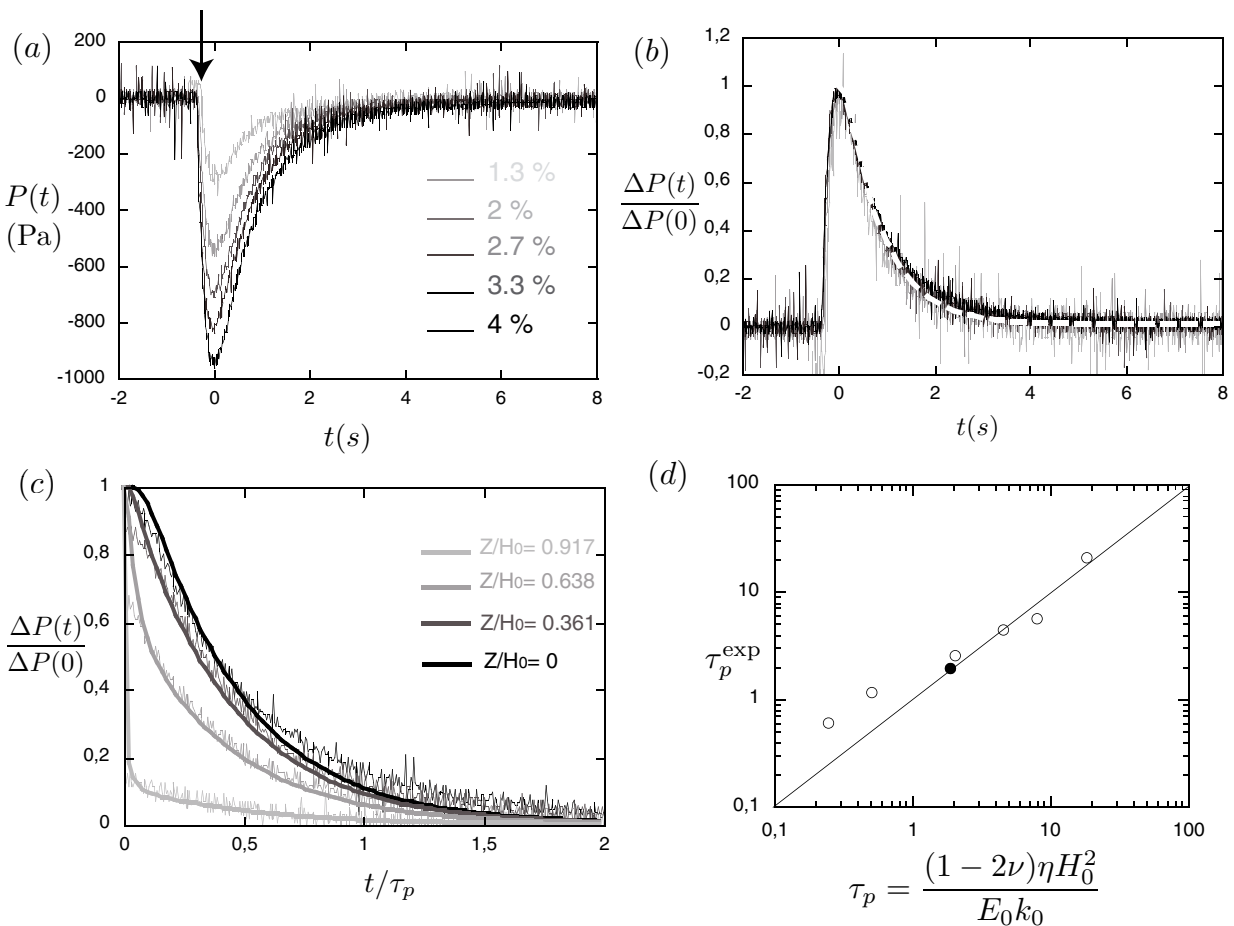

Fig. 2. Poroélasticité linéaire. (a) Pression interstitielle en fonction du temps mesurée sous la mousse au centre $(z=0)$, pour différentes compressions initiales $\left(H_{0}=12 \mathrm{~cm}, \eta=0,104 \mathrm{~Pa} . \mathrm{s}\right)$. La flèche correspond à la suppression de la contrainte extérieure. (b) Mêmes données normalisées par l'amplitude de la dépression initiale. Le fit en train blanc pointillé correspond à la solution de l'équation de diffusion (2) pour $\tau_{\mathrm{p}}=1,97 \mathrm{~s}$. (c) Champ de pression expérimental et théorique pour 4 positions verticales (la surface libre correspond à $z / H_{0}=1$ ). (d) Temps poroélastique pour différentes hauteurs de mousse $1,2<H_{0}<12 \mathrm{~cm}$ et viscosité (rond noir $\eta=0,104$ Pa.s, ronds blancs $\eta=1$ Pa.s).

supprimée (pointillé), on constate que la pression fluide à la base de la mousse décroît brutalement, avant de relaxer exponentiellement vers sa valeur initiale d'équilibre (Fig. 2a, les pressions sont par la suite toujours mesurées par rapport à l'état de référence d'équilibre hydrostatique). Des expériences menées pour différentes compressions initiales montrent que les courbes de relaxation de pression se superposent quand on les normalise par la dépression initiale (Fig. $2 \mathrm{~b}$ ). La réponse du système est donc linéaire dans ce régime.

Il est possible de comparer ces résultats aux prédictions de la poroélasticité linéaire [3]. Dans notre configuration d'écoulement unidirectionnel, en supposant les grandeurs dépendantes de $z$ uniquement et les contraintes latérales nulles (bords libres), on peut montrer que la pression de pore vérifie une équation de diffusion linéaire avec pour conditions aux limites : (i) pression interstitielle nulle à la surface libre, (ii) flux nul à la base de la mousse. La condition initiale à $t=0$ stipule que la pression interstitielle est égale à la contrainte élastique uniforme $p(z, 0)=p_{\mathrm{i}}=E_{0} \epsilon_{\mathrm{i}}$. Avec ces conditions, le champ de pression est donné par :

$$
\begin{aligned}
& p(z, t)=\sum_{n \geqslant 0} \frac{2 p_{i}}{H_{0} k_{n}} \sin \left(k_{n} z\right) e^{-t / \tau_{n}} \\
& \quad \text { avec } \tau_{n}=\frac{\tau_{\mathrm{p}}}{\left(\frac{\pi}{2}+n \pi\right)^{2}}, k_{n}=\left(\frac{\pi}{2 H_{0}}+\frac{n \pi}{H_{0}}\right)
\end{aligned}
$$

où

$$
\tau_{\mathrm{p}}=\frac{(1-2 \nu) \eta H_{0}^{2}}{E_{0} k_{0}}
$$

est le temps poroélastique, $\nu$ le coefficient de Poisson (drainé), $E_{0}$ le module de Young de la mousse (drainée), $k_{0}$ la perméabilité hydraulique et $\eta$ la viscosité du fluide.

Les figures $2 \mathrm{~b}$ (insert) et c comparent l'expérience et la solution de l'équation de diffusion. L'ajustement est bon et prédit un temps poroélastique $\tau_{\mathrm{p}}^{\exp }=1,95 \mathrm{~s}$ proche du temps théorique $\tau_{\mathrm{p}}^{\text {theo }}=(1-2 \nu) \eta H_{0}^{2} /\left(E_{0} k_{0}\right)=1,90 \mathrm{~s}$ prédit à partir des valeurs du module de Young, du coefficient de Poisson et de la perméabilité du milieu (voir Sect. 2 Matériels et Méthodes). La dépendance en $H_{0}^{2}$ du temps poroélastique est également assez bien vérifiée expérimentalement, sauf pour les plus petites hauteurs (Fig. 2d).

\subsection{Dynamique non-linéaire : fronts de décompaction}

\subsubsection{Phénoménologie}

Nous avons vu que dans le régime de compression linéaire, la décompaction de la mousse dans le fluide suit une dynamique diffusive, bien prédite par la poroélasticité classique. Que se passe-t-il quand on comprime la mousse dans le domaine non-linéaire de la loi de comportement (plateau de contrainte)? La figure 3a montre le champ de 

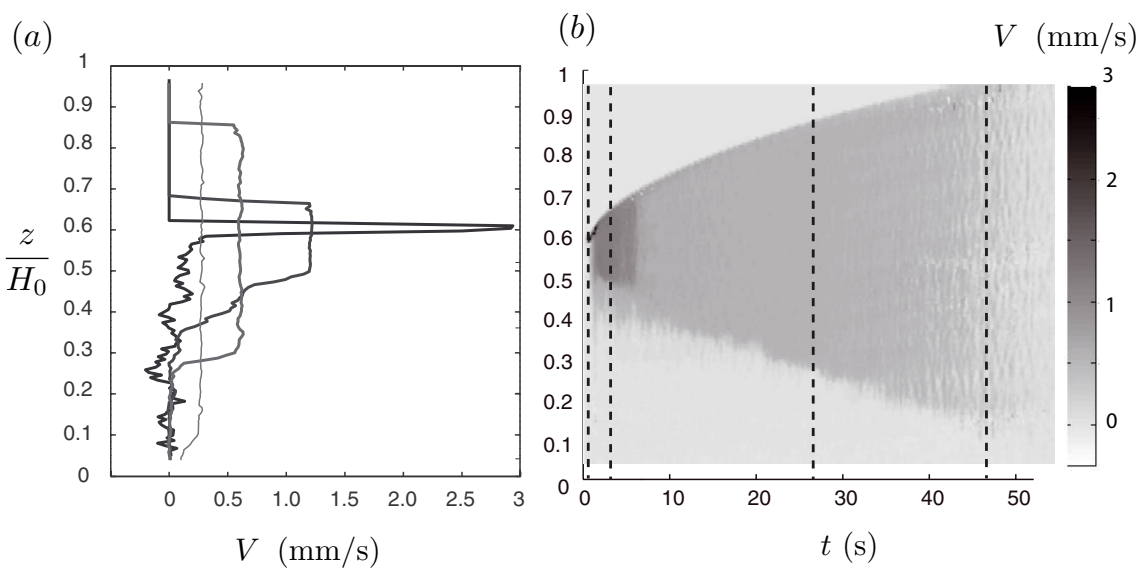

Fig. 3. Poroélasticité en grande déformation $\left(\epsilon_{i}=40 \%, H_{0}=8 \mathrm{~cm}, \eta=1,0\right.$ Pa.s). (a) Champ de vitesse spatiale dans la mousse mesuré par PIV à 4 instants différents (pointillés noirs sur b). (b) Diagramme spatio-temporel du champ de vitesse de la mousse $V(z, t)$.

vitesse verticale dans la mousse mesuré par PIV lors de la décompaction d'une mousse de hauteur $H_{0}=8 \mathrm{~cm}$ initialement comprimée à $\epsilon_{\mathrm{i}}=0,4$ dans un fluide de viscosité $\eta=1$ Pa.s. Aux premiers instants, on observe un pic de vitesse au niveau de la surface libre. Ensuite, le champ de vitesse verticale de la mousse présente trois zones : une première zone de vitesse quasi-constante au niveau de la surface libre; une zone où la vitesse décroit rapidement et enfin une zone de vitesse quasi-nulle au niveau du fond. Contrairement à la dynamique diffusive du régime linéaire, la décompaction se fait donc dans une zone localisée entre deux zones de vitesse constante. Ce front de décompaction est bien mis en évidence dans le diagramme spatio-temporel de la figure $3 \mathrm{~b}$. Le front se propage à vitesse quasi-constante de la surface libre vers le fond. Cette dynamique fortement non-linéaire est également répercutée sur le signal de pression de pore (Fig. 4c). La relaxation de pression présente une dynamique en deux étapes avec deux temps caractéristiques bien distincts : un premier temps de relaxation long $\tau_{N L}$, suivi d'un temps rapide de l'ordre du temps poroélastique $\sim \tau_{\mathrm{p}}$.

\subsubsection{Modélisation}

Pour tenter de comprendre la dynamique observée, nous utilisons une approche diphasique dans laquelle la mousse et le fluide interstitiel sont modélisés par deux phases continues incompressibles qui s'interpénètrent [10]. Nous supposons le problème unidirectionnel. La conservation de la masse pour la phase solide (mousse élastique) et pour le mélange (mousse + fluide) s'écrit :

$$
\frac{\partial \phi}{\partial t}+\frac{\partial \phi V}{\partial z}=0 \quad \text { et } \quad \phi V+(1-\phi) v=0
$$

où $\phi$ est la fraction volumique solide, $V$ la vitesse de la mousse et $v$ la vitesse du fluide. En l'absence d'inertie $(R e \ll 1)$, la conservation de la quantité de mouvement pour le mélange s'écrit simplement :

$$
\frac{\partial}{\partial z}(\sigma(\phi)+p)=0
$$

où $\sigma(\phi)$ est la loi de comportement en compression (en l'absence de fluide) de la mousse avec $\epsilon=1-\left(\phi_{0} / \phi\right)\left(\phi_{0}=\right.$ 0,03 est la fraction volumique de la mousse au repos) et $p$ la pression du fluide interstitiel. Enfin, la conservation de la quantité de mouvement pour la phase fluide se réduit à la loi de Darcy dans le référentiel du solide [10] :

$$
v-V=-\frac{k(\phi)}{\eta(1-\phi)} \frac{\partial p}{\partial z}
$$

où $\eta$ est la viscosité du fluide et $k(\phi)$ la perméabilité hydraulique de la mousse en fonction de la fraction volumique. En combinant les équations (4)-(10), on trouve une équation de diffusion non-linéaire pour la fraction volumique solide de la mousse :

$$
\frac{\partial \phi}{\partial t}=\frac{\partial}{\partial z}\left(D(\phi) \frac{\partial \phi}{\partial z}\right) \quad \text { avec } \quad D(\phi)=\frac{\phi k(\phi) \sigma^{\prime}(\phi)}{\eta}
$$

où $\sigma^{\prime}(\phi)=\mathrm{d} \sigma / \mathrm{d} \phi$. Cette équation est complétée par les conditions aux limites :

$$
\begin{array}{r}
\phi(H(t))=\phi_{0}, \frac{\mathrm{d} H}{\mathrm{~d} t}=V(H)=-\frac{D\left(\phi_{0}\right)}{\phi_{0}} \frac{\partial \phi}{\partial z}_{z=H} \\
\text { et } \frac{\partial \phi}{\partial z}=0
\end{array}
$$

Dans notre cas, la loi de comportement $\sigma(\epsilon)$ peut être modélisée comme la succession de deux pentes de modules de Young $E_{1} \sim 10 E_{2}$, la transition ayant lieu pour $\epsilon_{\mathrm{c}} \sim 5 \%$ (Fig. 4a). Le coefficient de diffusion $D(\phi) \sim$ $k(\phi) E \phi_{0} / \eta \phi$ prend donc deux valeurs très différentes $D_{1} \gg D_{2}$ selon la valeur de $\phi$ (Fig. 4a). Dès lors, on s'attend à ce que la dynamique de relaxation de la mousse soit décomposée en deux zones. Pour $\phi<\phi_{\mathrm{c}}$, la diffusion est très rapide et le milieu atteint rapidement un régime pseudo-stationnaire où la déformation passe linéairement de 0 (à la surface libre) à $\phi_{\mathrm{c}}$. La vitesse de la mousse dans cette zone est quasi-constante et donnée par la loi de Darcy et la conservation de la masse. Pour $\phi>\phi_{\mathrm{c}}$ au 
(a)

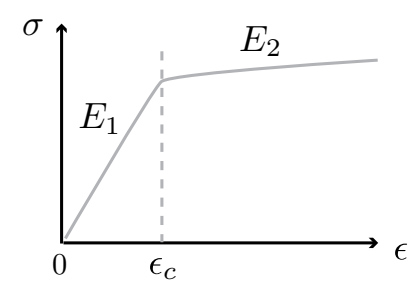

$(b)$

$(c)$
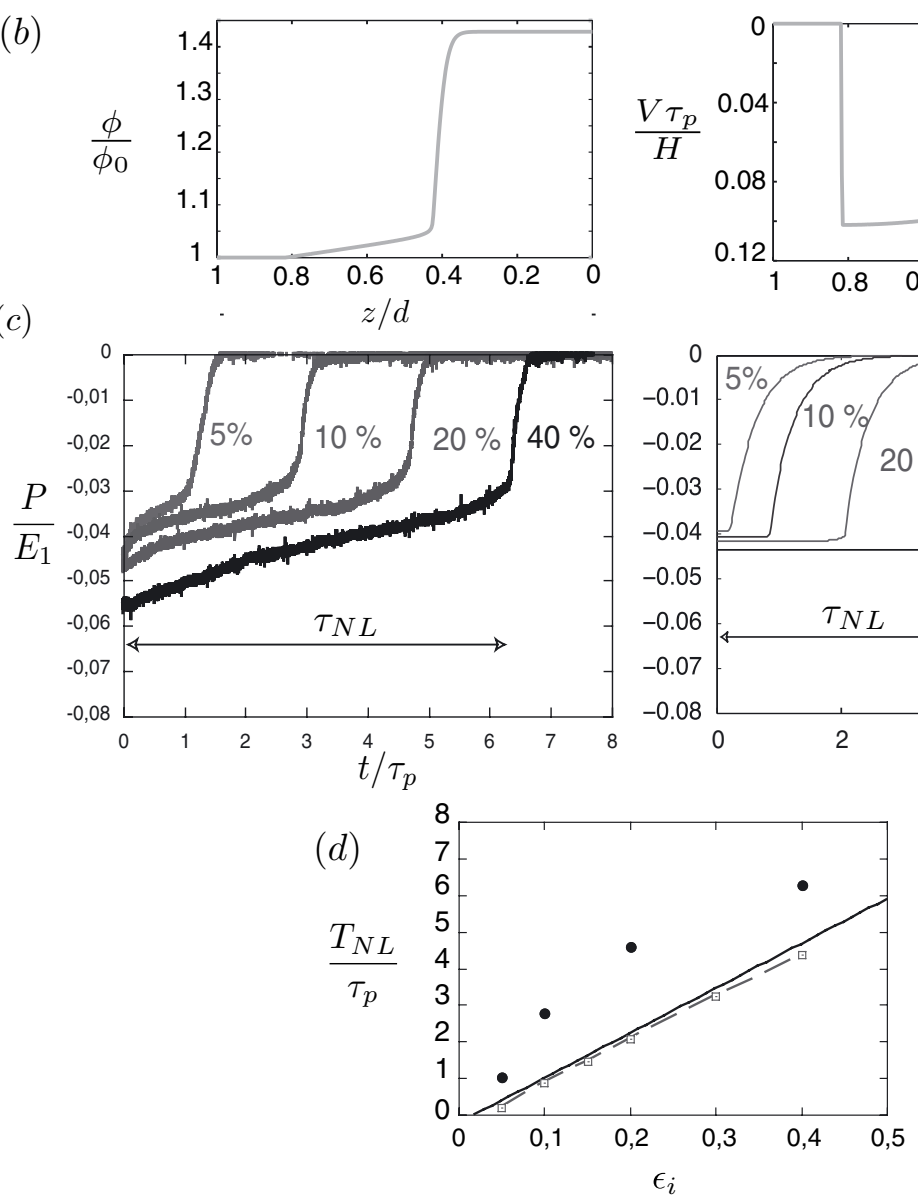
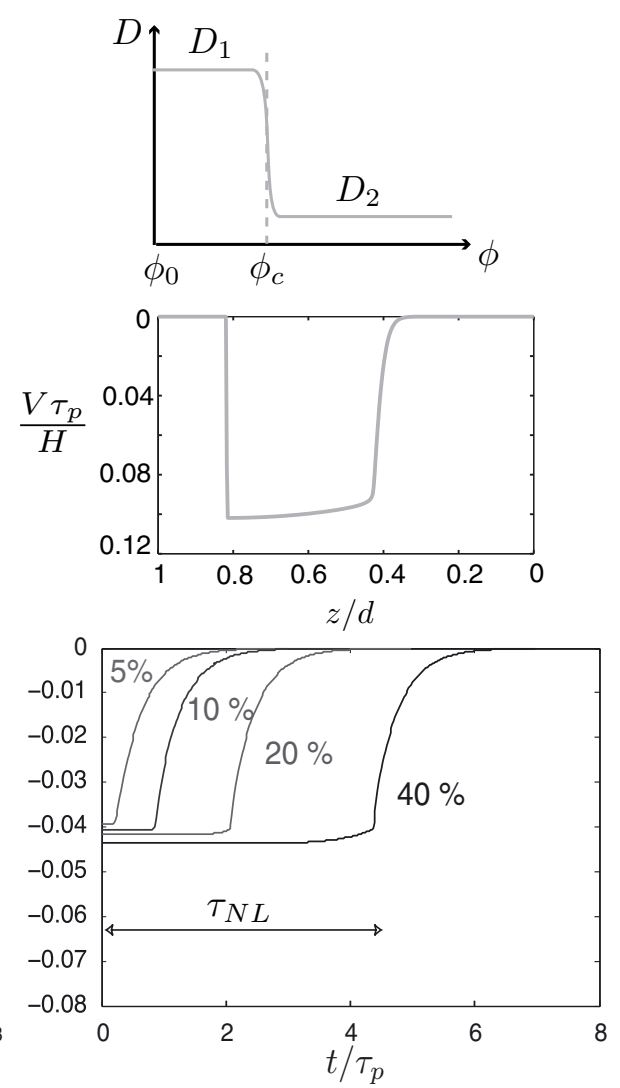

Fig. 4. (a) Modélisation de la loi de comportement de la mousse introduite dans le modèle et coefficient de diffusion effectif associé. (b) Profils de concentration et de vitesse typique lors de la décompaction obtenus par simulation numérique du modèle diphasique $\left(\epsilon_{\mathrm{c}}=4 \%, \epsilon_{\mathrm{i}}=40 \%, E_{2} / E_{1}=0,01\right)$. (c) Comparaison expérience/modèle pour la relaxation de pression. (d) Temps de décompaction de la mousse $\tau_{\mathrm{NL}}$ en fonction de la compaction initiale dans l'expérience (ronds) et le modèle (carrés, $E_{2} / E_{1}=0,01, k(\phi)=$ cste). Le trait continu est l'expression analytique (9) prédite dans le cas $D_{1} \gg D_{2}$.

contraire, la diffusion est très lente et l'on a $V \approx 0$ et $\phi \approx \phi_{i}$ (la compacité initiale de la mousse). La transition entre les deux zones est localisée autour d'un front $z=h(t)$, dont la vitesse est imposée par la conservation de la masse et par la continuité du «flux $» D(\phi) \partial \phi / \partial z$.

\subsubsection{Prédiction et comparaison avec l'expérience}

Une résolution numérique complète du problème (7)(8) dans le cas $E_{2} / E_{1}=0,01$ et $k(\phi)=$ cste confirme ce scénario (Figs. 4b,c). Les profils de vitesse et de concentration sont proches de ceux prédits par l'analyse asymptotique précédente et compatibles avec les observations expérimentales (Fig. 4b). De même, on retrouve une relaxation de pression qui présente deux temps distincts comme dans l'expérience (Fig. 4c).

$\mathrm{Si}$ le modèle reproduit bien les principales caractéristiques de la dynamique de décompaction observée, il existe des différences quantitatives entre théorie et expérience. Le principal désaccord concerne le temps total de décompaction (Fig. 4d). Dans le modèle, le temps de décompaction reste contrôlé par le temps poroélastique $\tau_{\mathrm{p}}=\eta H^{2} /\left(E_{1} k\right)$ comme dans le régime linéaire, et dépend peu du module de Young $E_{2}$ de la partie non-linéaire. On peut en particulier montrer que dans la limite $D_{1} \gg D_{2}$, correspondant à $E_{1} \gg E_{2}$, le temps de décompaction varie comme :

$$
\tau_{\mathrm{NL}}^{\mathrm{asymp}} \approx\left[\left(\phi_{i}-\phi_{0}\right) /\left(2 \epsilon_{\mathrm{c}} \phi_{i}\right)\right] \tau_{p}
$$


indépendamment de la valeur de $D_{2}$ (Fig. 4d). Expérimentalement en revanche, le temps de décompaction est plus long et semble contrôlé par le module de Young le plus faible $\left(E_{2}\right)$ (Fig. 4d, ronds noirs). Notons que l'introduction dans le modèle d'une loi de comportement $\sigma(\epsilon)$ non idéalisée comme sur la figure 4a et ajustée aux données expérimentales (Fig. 1a) ne permet pas de rapprocher le temps de décompaction prédit de celui mesuré expérimentalement. De même, la prise en compte de la dépendance de la perméabilité hydraulique avec la fraction volumique $k(\phi)$ (Fig. 1a) ne modifie pas significativement le temps de décompaction prédit.

Plusieurs pistes peuvent être proposées pour expliquer cet écart entre prédiction et expérience. La première concerne le rôle du confinement latéral de la mousse et d'un éventuel frottement aux parois qui ralentirait la dynamique de décompaction. On peux estimer les forces de frottement de type visqueuse entre la mousse et les parois latérales comme :

$$
F_{\text {frot }}^{\mathrm{visc}} \approx \eta(V / \delta) 4 a H_{0} \phi_{s} \approx k(P / \delta) 4 a \phi_{s}
$$

où $\delta$ est la distance entre la mousse et la paroi et $\phi_{s}$ la fraction surfacique de mousse solide à la paroi. En prenant $k=5 \times 10^{-9} \mathrm{~m}^{2}, \phi_{s} \approx \phi^{\frac{2}{3}} \approx 0,1, a=8 \mathrm{~cm}$, on trouve que cette force reste très petite devant la force élastique dans la mousse $F_{\text {el }}=\sigma a^{2}=P a^{2}$ tant que la distance mousse/paroi vérifie $\delta \gg 0,3 \mu \mathrm{m}$. Dans notre expérience, cette condition est largement remplie dans le régime de faibles déformations $(\delta \sim \mathrm{mm})$. En revanche, pour des grandes déformations, on observe une ondulation de la mousse et des points de contact avec la paroi peuvent apparaître. Pour tenter de s'affranchir de ce frottement éventuel aux parois, des expériences en milieu non confiné sont en cours sur des mousses à parois latérales étanches.

La deuxième possibilité pour expliquer le désaccord théorie/expérience est plus profonde. Dans le modèle, nous avons modélisé la mousse comme un milieu continu dans lequel les déformations sont homogènes. En réalité, pour $\epsilon>\epsilon_{\mathrm{c}}$, les déformations dans la mousse sont fortement inhomogènes, et des zones de fortes compacités locales (les cellules ont flambé) coexistent avec des zones peu compactées [7,9]. Lors du passage du front de décompaction, il est possible que ces zones fortement compactées, dans lesquelles les brins solides sont en « contact », offrent une résistance élevée en raison des forces de lubrification visqueuse entre les brins solides de la mousse. Ces forces de lubrification ne sont pas prises en compte dans les équations diphasiques classiques [10], dans lesquelles la force d'interaction entre la phase solide (la mousse) et la phase liquide est donnée par une simple loi de Darcy (Éq. (10)). Nous comptons explorer cette question à la fois d'un point de vue théorique (construction d'un modèle continu avec force de lubrification) et expérimental (mise en place d'une mesure locale du champs de déformation à l'échelle de la cellule par méthode optique dans un fluide iso-indice).

\section{Conclusion}

Notre étude a mis en évidence l'existence de front de décompaction dans des mousses poroélastiques fortement déformées. Cette dynamique est reliée à la réponse mécanique fortement non-linéaire de ce matériau cellulaire. Il est intéressant de remarquer qu'il existe formellement une analogie entre notre système et l'évaporation d'une solution de polymères près de la transition vitreuse. Dans ce cas, au niveau de la surface libre, la concentration en polymères est élevée et dépasse la transition vitreuse, ce qui augmente fortement le coefficient de diffusion du solvant (le module « élastique » est grand). Il peut alors se former une « croûte » d'épaisseur nanométrique au niveau de la surface libre [11,12], qui est analogue à notre région de vitesse constante près de la surface libre de la mousse.

\section{Références}

[1] K. Terzaghi, Theoretical soil mechanics, John Wiley New York, 1943

[2] M.A. Biot, General theory of three-dimensional consolidation, J. Appl. Phys. 12 (1941) 155-165

[3] H.F. Wang, Theory of linear poroelasticity with applications to geomechanics and hydrogeology, Princeton University, 2000

[4] O. Coussy, Poromechanics, Wiley, 2003

[5] G.W. Scherer, Bending of gel beams: Effect of deflection rate and Hertzian indentation, J. Crys. Solids 201 (1996) $1-25$

[6] P. Hebraud, F. Lequeux, J. Palierne, Role of permeation in the linear viscoelastic response of concentrated emulsions, Langmuir 16 (2000) 8296-8299

[7] M.A. Dawson, G.H. McKinley, L.J. Gibson, The dynamic compressive response of open-cell foam impregnated with a newtonian fluid, J. Appl. Mech. 75 (2008) 041015

[8] H.-C. Tsai, Compression analysis of rectangular elastic layers bonded between rigid plates, Int. J. Solid Struct. 42 (2005) 3395-3410

[9] L.J. Gibson, M.F. Ashby, Cellular solids, Cambridge University Press Cambride, 1999

[10] R. Jackson, The dynamics of fluidized particules, Cambridge University Press, 2000

[11] P.-G. de Gennes, Solvent evaporation of spin cast films: "crust" effects, Eur. Phys. J. E 7 (2002) 31

[12] T. Okuzono, K. Ozawa, M. Doi, Simple model of skin formation caused by solvent evaporation in polymer solutions, Phys. Rev. Lett. 97 (2006) 136103 